\title{
Le risque d'accident nucléaire : prévention et gestion
}

Entretien croisé(a) avec Jean-Christophe Gariel, Institut de Radioprotection et de Sureté Nucléaire,

et Sophia Majnoni d'Intignano, juriste, ancienne chargée de mission nucléaire à Greenpeace France

\section{Estimer le risque d'un accident nucléaire, et s'y préparer, comporte de grandes difficultés et des hypothèses qui font rarement consensus. Quant à la gestion d'un éventuel accident, l'Histoire a montré que les décisions et choix opérationnels dépendent de la structure politique et sociale des territoires concernés. Regards croisés de deux experts du domaine.}

Avant la catastrophe de Tchernobyl, l'évaluation de la gravité des accidents nucléaires était laissée à l'appréciation de l'exploitant. Après Tchernobyl, une échelle internationale des évènements nucléaires, appelée INES, a été créee pour définir et classer les incidents et accidents nucléaires. L'accident concerne les réacteurs, les piscines de combustibles, ainsi que les centres de stockage de déchets (comme celui de Maïak à Kychtym, en Russie, également centre de production de plutonium, et siège en 1957 d'un important accident peu connu du grand public).

Les principaux risques identifiés sur les installations nucléaires civiles françaises sont liés notamment aux piscines de la Hague, très remplies ; au vieillissement des équipements de production ; et au risque d'attentat sur un point de la chaine. En outre, pour les transports (rendus plus nombreux par le choix du retraitement), la France a fait le choix du camion : cela permet à EDF de traiter entièrement le transport en interne, avec donc un meilleur contrôle de sécurité de toute la chaine, mais les risques d'accident de camion sont plus élevés que ceux du train.

\section{La prise en compte des risques}

Les risques rares et importants sont délicats à évaluer, et ils sont également difficiles à prendre en compte lors des prises de décision, tant pour les experts que pour l'opinion publique. De plus, dans le cas du nucléaire, les effets des radiations à faible dose ne sont pas visibles à l'œil nu ni directement inclus dans notre intuition quotidienne. Enfin, le débat sur le risque est entaché par le manque de consensus sur l'effet de l'exposition à des faibles doses de radioactivité, et le lien par exemple avec les maladies professionnelles des travailleurs du nucléaire. Cela concerne toute la chaine, y compris hors accident, pour les étapes en amont telle que l'extraction minière, ou autour des centres de stockage.

Les décisions politiques et la préparation aux accidents nécessitent de prendre en compte correctement le risque. Or l'approche probabiliste, développée pour les évènements répétitifs et assurables, est moins consensuelle quand elle s'applique aux risques extrêmes et rares; elle suscite depuis longtemps un désaccord profond entre les militants antinucléaires et les autorités de contrôle. L'exemple par excellence est le risque de chute d'avion. En outre, un accident niveau 7 de l'échelle INES au milieu d'un désert pourrait être plus acceptable politiquement et socialement, mais pas écologiquement, qu'un accident niveau 6 à la centrale d'Indian Point à moins de 40 kilomètres de la ville de New York. Un système international d'assurance du risque a été mis en place ; il oblige chaque réacteur à être assuré à hauteur de 750 millions d'euros et les États à prendre la relève jusqu'à 1,5 milliard. C'est à comparer au cout d'une catastrophe du type de celle de Fukushima, estimé par la Cour des comptes à plusieurs centaines de milliards d'euros ${ }^{(b)}$.

\section{La prévention des risques}

La prévention des risques fait débat sur plusieurs points. Ainsi, l'accident de Fukushima a montré que les dispositifs de confinement, bien que redondants, pouvaient être mis en défaut. Le choix de stocker sous terre des déchets de haute activité à demi-vie de plusieurs milliers d'années soulève de difficiles questions d'anticipation, en particulier des risques sismiques ou politiques. Sophia Majnoni d'Intignano souligne que cela pose des problèmes moraux vis-à-vis des générations futures; vaut-il mieux éviter de signaler le centre de stockage, voire miser délibérément sur l'oubli, ou bien tenter de leur communiquer (comment ?) son existence et les dangers associés ${ }^{(c)}$ ?

Il est nécessaire de maintenir des travailleurs compétents, et ceci sur des décennies ou même plus (cela vaut aussi bien pour la maintenance et le démantèlement, que pour la préparation à un éventuel accident). Or, les différents éléments de l'industrie nucléaire sont imbriqués ; toute décision, même partielle, peut avoir des répercussions à long terme sur l'ensemble. Par exemple, même s'il y a d'importants désaccords sur la date, il faudra bien un jour fermer les 15 réacteurs anciens, de 900 MW. Comme ce sont les seuls qui utilisent le combustible « Mélange d'oxydes d'uranium et de plutonium " (MOX), leur fermeture aura un impact sur l'usine Melox de Marcoule qui le fabrique et sur l'usine de retraitement de La Hague qui en fournit le plutonium ; et plus généralement sur leur industrie, leurs 5000 emplois, et le savoir-faire de la filière.

Pour Sophia Majnoni d'Intignano, la solution réside peut-être dans l'approche des Allemands, dont le comité d'éthique intègre une projection dans le long terme : on peut donner une valeur morale, intellectuelle ou sociétale au fait de gérer le passif du nucléaire, au bénéfice des 

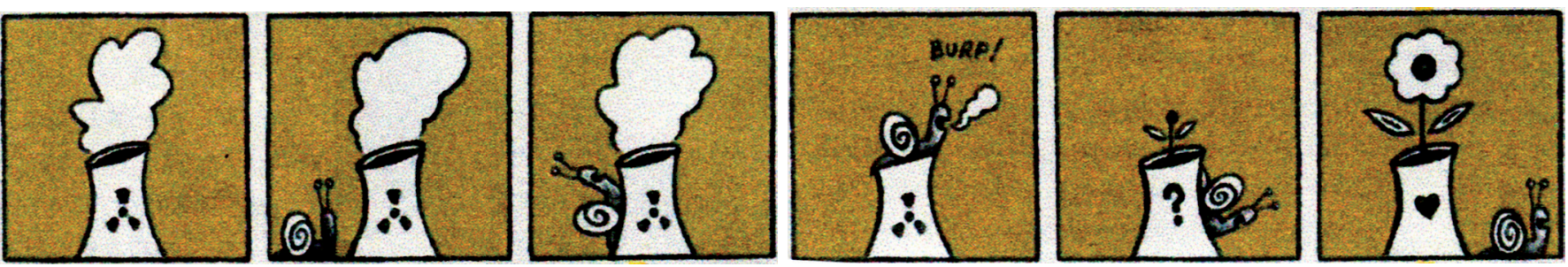

générations futures. C'est une manière, alternative ou complémentaire au développement de nouveaux programmes nucléaires, de garder à long terme une dynamique positive et d'attirer les personnes compétentes, pour la décontamination et le démantèlement (qui nécessiteront aussi des capacités de financement et des réserves d'énergie).

\section{La préparation à un éventuel accident}

Des dispositions sont préparées aux niveaux local, national, voire international pour être mises en œuvre en vue de faire face aux situations d'accident. Au Japon le risque et sa gestion sont des sujets très importants, notamment en raison de la fréquence des tremblements de terre, et les écoliers y sont tous sensibilisés ; après Fukushima, la cohésion sociale du Japon a permis une évacuation de la zone contaminée qui ne se serait peut-être pas aussi bien déroulée dans un autre groupe social.

Pour limiter la panique en cas d'accident, il est important de former les collectivités (pour le transport des habitants, l'hébergement temporaire ou l'information), les pompiers, les pharmacies, etc. JeanChristophe Gariel souligne que les dispositions de protection des personnes du public visent à limiter leur exposition radiologique à un niveau aussi faible que raisonnablement possible. La prise d'iode stable est utile en cas de rejet contenant de l'iode radioactif (ce qui peut être le cas pour un accident de réacteur nucléaire par exemple). Elle vise, par une saturation précoce de la glande thyroïde, à limiter la fixation d'iode radioactif dans cette glande. En France, actuellement, ce sont les personnes habitant à moins de $15 \mathrm{~km}$ d'un réacteur nucléaire (zone dite " périmètre particulier d'intervention ") qui sont spécifiquement informées sur le risque nucléaire, qui participent à un exercice nucléaire et qui peuvent trouver dans leur pharmacie des comprimés d'iodure de potassium. Il pourrait être utile d'étendre ces zones, sachant que 75\% des Français de métropole habitent à moins de $75 \mathrm{~km}$ d'un réacteur nucléaire; pour eux, la distribution d'iode est réalisable à partir des stocks départementaux.

Certains accidents peuvent nécessiter une réaction très rapide. Des rejets importants et de courte durée peuvent conduire à demander aux habitants de se mettre à l'abri dans des bâtiments ; JeanChristophe Gariel rappelle que cela réduit à la fois l'irradiation externe et le risque d'inhaler un air contaminé. Ce type de mesure peut être envisagé pendant une douzaine d'heures ; voire pendant plusieurs jours si elle a été préparée dans de bonnes conditions, mais l'air risque alors d'être aussi contaminé à l'intérieur des bâtiments. On peut cependant s'interroger sur le degré d'obéissance des personnes concernées : par exemple, qui accepterait de laisser ses enfants confinés à l'école ?

Les actions de protection à prendre en urgence sont définies à l'avance et dépendent de la situation. La décision de prise d'iode stable est diffusée par les médias, en précisant quand et comment la prendre, quelles sont les personnes concernées et celles qui sont prioritaires (enfants et femmes enceintes en particulier). En parallèle, des actions de sécurité publique (par exemple une restriction de circulation sur les voies publiques) et de maintien de l'ordre sont mises en œuvre. Le préfet peut décider de restreindre la consommation de certaines denrées ou certaines activités particulières. Enfin, l'évacuation de la zone peut être décidée, auquel cas les pouvoirs publics devront prendre en charge les personnes non autonomes.

\section{La phase postaccidentelle}

Dans la phase postaccidentelle, les questions touchant à la qualité de l'environnement, à la santé des populations, à la continuité de la vie sociale et économique ainsi qu'aux relations internationales sont des sujets majeurs. Ils concernent cette fois le niveau national, et la prise de décision peut associer étroitement les différentes parties prenantes, en premier lieu les habitants des zones touchées. Comment définir la taille des périmètres restreints, voire complètement interdits ? Vaut-il mieux laisser une population vivre au contact de doses de radioactivité supérieures aux normes (et jusqu'où), ou bien fermer la zone en dispersant les familles et en arrêtant toute activité ?

Le débat sur l'effet des faibles doses d'irradiation a alors des conséquences pratiques de grande ampleur. Il y a des territoires faiblement contaminés qu'on souhaite habiter pour des raisons sociales, humaines, économiques. Or au-dessous d'une certaine dose, on ne sait pas évaluer des effets statistiques significatifs, et ce n'est pas non plus fiable d'extrapoler aux faibles doses ce que l'on sait sur les effets des doses moyennes et fortes. Si on renonce à définir un seuil, le principe qui guide l'action politique revient à minimiser, quoi qu'il arrive, la dose de radioactivité que l'on permet d'ajouter à la dose ambiante, en agissant sur les comportements (alimentation, horaires) et en favorisant les mesures de radioactivité par les habitants concernés eux-mêmes. Le politique doit arbitrer les différentes mesures en pesant tous les risques et impacts : environnemental, social et économique.

a.Voir dans ce dossier le préambule aux entretiens croisés, par F. Graner et S. M. Panebianco (p. 18)

b.Voir dans ce dossier l'article d'A.-S. Dessillons (p. 29).

c. Olivier Le Naire, "Enfouissement des déchets nucléaires : comment alerter nos descendants ? ", lexpress.fr, 8 novembre 2014, www.lexpress.fr/actualite/sciences/enfouissement-des-dechets-nucleaires-commentalerter-nos-descendants_1619017.html 